\title{
A COMPARATIVE TRIAL OF OIL-ADJUVANT AND AQUEOUS POLYVALENT INFLUENZA VACCINES
}

\section{BY}

\author{
R. W. HOWELL and A. B. MACKENZIE
}

From the Authority Health and Safety Branch, Harwell, and the Medical Department, Risley, U.K. Atomic Energy Authority

(RECEIVED FOR PUBLICATION AUGUST 19, 1964)

In the early autumn of 1963, 957 volunteers were inoculated with Admune (an oil-adjuvant polyvalent influenza vaccine) and 337 with Munevan (an aqueous polyvalent vaccine).

A survey by questionnaire suggested that the oil-adjuvant vaccine was more acceptable to the inoculated than the aqueous one. The oil-adjuvant vaccine seemed less likely than the aqueous to provoke serious side effects in unknown reactors, and this more than offset the possibility of subcutaneous nodules not encountered with the aqueous vaccine. No difficulty was encountered in administering the oil-adjuvant vaccine, in the recommended dose, to those giving a history of hay fever, asthma, and some other major allergies.

On this occasion, reactions to both vaccines were fairly prevalent, and about a quarter of all those vaccinated complained of a feeling of malaise lasting at least two weeks. These reactions were thought to be severe enough to induce consumer-resistance to routine annual immunization, which is considered hardly acceptable in a healthy industrial population at the present stage of development. Medically the case for such immunization is not yet proven.

There is a need for further vaccine development and for more controlled trials. If the efficacy of the oil-adjuvant vaccine is proved in clinical practice, this vaccine appears preferable to the aqueous type.

In addition to the results already reported in the previous paper (Howell and Stott, 1964), a comparative trial of oil-adjuvant and aqueous vaccines was carried out at the Authority's establishment at Risley, where 957 employees were inoculated with Admune (oil-adjuvant) and 337 with Munevan (aqueous) polyvalent vaccines. ${ }^{*}$ It was essential that identical strains were used in the two vaccines, and this was assured by courtesy of Evans Medical Ltd. who kindly supplied the vaccines, which were made from a common source of virus concentrate.

Admune was administered as a single dose of $0.25 \mathrm{ml}$. and Munevan was given in a $1 \mathrm{ml}$. single dose. The vaccines were administered intramuscularly into one or other of the deltoid muscles.

As reported in the previous paper, no significant protection could be demonstrated against clinical influenza in a non-epidemic year, and the main interest lies in the reactions encountered, and the acceptability of the vaccines by those inoculated.

\footnotetext{
- The methods by which the comparability of the groups were assessed are available in an Authority report (AHSB (RP) R46) (Howell, R. W., and Mackenzie, A. B., 1964).
}

\section{Reactions}

The few subjects who gave a history of allergy were excluded from the trial, but no difficulties were encountered in their vaccination with Admune. One Munevan subject, who gave a history of bronchitis only, collapsed immediately after injection with an asthma-type reaction, and adrenaline had to be administered before he made an uneventful recovery. Two Admune patients developed subcutaneous nodules. This tendency to nodulation was pointed out by Salk and emphasizes the need for deep intramuscular injections.

In June 1964 - nine months after inoculation-a questionnaire was sent to all those who had been vaccinated. One object of this late enquiry was to ascertain whether those inoculated would volunteer again if protection were offered. Obviously this question was better answered after a full winter at risk, rather than shortly after inoculation. There was a danger that the original symptoms might be difficult to recall after such an interval. Conversely, 
the minor inconvenience, for most people, of vaccination, should have fallen into perspective. Only 14 replies were rejected because of ambiguity or incompleteness. In a few cases the length of time before symptoms commenced could not be recollected, but these replies were accepted. After allowance for staff who had left or died, and those known to be on long-term sickness absence or maternity leave, there was a remarkably good response. Although replies were likely to be a biased sample of those vaccinated, those having strong feelings about the reactions being more likely to return a questionnaire than the others, and those who had no firm ideas about future acceptance or rejection of protection being probably less inclined to reply, a return of $86 \%$ for Admune and $80 \%$ for Munevan was highly satisfactory.

In the questionnaire, details of four symptoms were specifically sought. These were (1) swelling and inflammation of the arm; (2) discomfort lasting more than 24 hours at the site of injection; (3) persistent influenza-like feelings; and (4) a raised temperature and influenza-like feeling soon after injection. In addition: a request was made for details (if any) of additional symptoms thought to be associated with the vaccine. A few cases of catarrh, sinusitis, and intermittent colds were reported, as were a few instances in which the vaccine was alleged to have caused an attack of hay-fever. These cases were not severe enough to be discernible in the sickness absence rates, and it seems probable that the incidence of catarrh and sinusitis was not dissimilar from that prevailing in the non-vaccinated group. For this reason, the analyses have disregarded symptoms other than the four prevalent ones, which are not mutually exclusive. It is of interest to note that, judging from the limited number who reported other symptoms when specifically invited to do so, the questionnaire did not induce people to exaggerate their reactions.

In the Admune group. $84.8 \%$ of those who returned questionnaires stated that they would again volunteer for vaccination, whereas the figure for the Munevan group was $80.5 \%$.

About half of those who replied stated that they had had no reaction, and, not surprisingly, the
TABLE 1

WILLINGNESS FOR RE-INOCULATION IN ALL STAFF CATEGORIES-QUESTIONNAIRE RESULTS

\begin{tabular}{l|c|c}
\hline & Admune & Munevan \\
\hline $\begin{array}{l}\text { Number without reaction } \\
\text { Willing for re-inoculation (\%) }\end{array}$ & 436 & 95.5 \\
\hline Number with reaction & 936 \\
Willing for re-inoculation (\%) & 71.0 & 122 \\
\hline
\end{tabular}

TABLE 2

INCIDENCE OF REACTIONS-QUESTIONNAIRE RESULTS

\begin{tabular}{l|c|c}
\hline \multirow{2}{*}{ Reaction } & Percentage with Reaction \\
\cline { 2 - 3 } & Admune & Munevan \\
\hline Swelling or inflammation of arm & $\mathbf{( 7 7 4 )}$ & $(258)$ \\
Discomfort (lasting more than & $\mathbf{7 . 5}$ & 18.6 \\
24 hr) at injection site & 15.6 & 17.8 \\
Persistent influenza-like feelings & $\mathbf{2 5 . 1}$ & $\mathbf{2 2 . 4}$ \\
Raised temperature and influenza- & 9.8 & 8.5 \\
like feelings soon after injection & 9.8 & 0 \\
\hline
\end{tabular}

percentage who agreed to re-inoculation was high in this group (Table 1).

Swelling or inflammation in the arm was considerably more frequent in the Munevan group (Table 2). Despite this, however, the proportions willing to undergo re-inoculation were very similar in both groups (Table 3 ).

Those complaining of persistent discomfort at the site of injection had moresevereclinicalsymptoms after the Munevan vaccination. It was not surprising, therefore, that the proportion willing for re-inoculation was very much less than in the Admune group (Table 3).

The symptom that was perhaps the most surprising was the persistent feeling of influenza or mild coryza lasting at least two weeks. This made the vaccines unacceptable to a higher proportion of staff than did other reactions. This malaise occurred in both groups with roughly equal frequency and severity. Of particular interest was the time after inoculation at which the symptoms began. Only about one person in five reporting this reaction found that it began within 48 hours, and between a third and a quarter did not experience symptoms until

TABLE 3

WILLINGNESS FOR RE-INOCULATION BY REACTION-QUESTIONNAIRE RESULTS

\begin{tabular}{|c|c|c|c|c|}
\hline \multirow{2}{*}{ Reaction } & \multicolumn{2}{|c|}{ Admune } & \multicolumn{2}{|c|}{ Munevan } \\
\hline & $\begin{array}{l}\text { No. with } \\
\text { Reaction }\end{array}$ & $\begin{array}{l}\% \text { Willing for } \\
\text { Re-inoculation }\end{array}$ & $\begin{array}{l}\text { No. with } \\
\text { Reaction }\end{array}$ & $\begin{array}{l}\% \text { Willing for } \\
\text { Re-inoculation }\end{array}$ \\
\hline $\begin{array}{l}\text { Swelling or inflammation of arm } \\
\text { Discomfort (lasting more than } 24 \mathrm{hr} \text { ) at injection site } \\
\text { Persistent influenza-like feelings } \\
\text { Raised temperature and influenza-like feelings soon after injection }\end{array}$ & $\begin{array}{r}58 \\
123 \\
194 \\
76\end{array}$ & $\begin{array}{l}69 \cdot 0 \\
80 \cdot 7 \\
62 \cdot 1 \\
71 \cdot 1\end{array}$ & $\begin{array}{l}48 \\
46 \\
58 \\
22\end{array}$ & $\begin{array}{l}66 \cdot 7 \\
63 \cdot 0 \\
61 \cdot 4 \\
63 \cdot 6\end{array}$ \\
\hline
\end{tabular}


more than a week after injection. This delayed reaction suggested toxicity of the antigenic content of the vaccine.

The fourth prevalent symptom, raised temperature and influenza-like illness almost immediately after injection, was overall the least common. Again Admune seemed to have been more acceptable.

There was no clear association between either age or staff category and reactions, but those aged 45 and over found inoculation (both vaccines) slightly more acceptable than those aged 15 to 44 years.

\section{Discussion}

In many years the acceptability of influenza vaccines may well depend on the cost of the treatment and the incidence and severity of the reactions. The critical factor in large-scale annual re-immunization may well be the acceptability of the vaccine to employees. Richardson and Kilpatrick (1964) found considerable ' consumer resistance' over a period of three consecutive years, particularly among women. Experience in the Authority (Meichen, Rogan, and Howell, 1962; Howell and Stott, 1964) has tended to confirm their findings. At Risley an attempt was made, by questionnaire, to confirm the clinical impression that the reactions, while generally mild, would affect the numbers accepting any future offer of protection.

It is difficult to frame a questionnaire that is both simple and free from criticism, but whatever the shortcomings of the present enquiry they were applicable to both groups, thus permitting a fair comparison. Although the response did not cover the complete sample, it was so good that it was thought that the analyses were realistic. After these analyses were made, less than four weeks after the issue of the questionnaire, replies continued to trickle in, and although no attempt was made to incorporate these late arrivals, it was noticed that about half of them reported no reactions and about $80 \%$ were willing for re-inoculation. This was roughly the position maintained among those who replied within a day or two of the issue of the questionnaire, and also among the later responses after two or three weeks. This tended to confirm the acceptability of the analyses.

Although the issue of a questionnaire nine months after inoculation may lead to the criticism that reactions would be difficult to remember, in practice few replies were rejected as unsatisfactory. It must also be borne in mind that after a winter singularly free from virus infection, the individual must decide whether or not to seek re-inoculation on an emotional rather than an objective basis. The recollection of past reactions, whether distinct or hazy, must be an important factor in this choice.

On the other hand, the percentage of those willing to accept re-inoculation would probably be smaller than that indicated here, since experience has shown that of those who volunteer for prophylactic vaccination (e.g., poliomyelitis) a proportion subsequently find some excuse for not attending. Nevertheless, this factor applied equally to both the Admune and Munevan groups, and did not invalidate direct comparison.

Although the advantage of a smaller dose would seem quite definite, in practice it was interesting to note that the acceptability of Admune was only slightly better than that of Munevan. To supplement this advantage, the likelihood of a serious reaction by previously unknown reactors appeared less in the Admune group, and it more than offset the possibility of the subcutaneous nodules not encountered with Munevan.

A persistent coryza or influenza-like feeling lasting at least two weeks was prevalent in both sexes, in manual and non-manual workers, at all ages, and was provoked by both vaccines in similar proportions. About $40 \%$ of those who reacted in this way showed no desire for re-vaccination. This widespread persistent feeling of malaise was unlikely to have been psychogenic and was probably associated with the vaccine processes. Since the two vaccines were identical in composition and these symptoms arose almost equally from both vaccines, it was unlikely to have been caused by the oil emulsifier, nor has such a widespread reaction been generally reported from aqueous vaccines. There was little likelihood of such a large sample of staff having been hypersensitive, so that the most probable cause was the virus composition of the vaccine. Whilst toxicity varies from strain to strain, the composition of a polyvalent vaccine must be changed from time to time to meet the expected influenza viruses, and this sort of reaction is likely to arise. The high proportion of reactors who reported the onset of symptoms more than 48 hours after inoculation also suggested an inherent toxicity in the virus content of the vaccine. Intensive testing of modified vaccines should therefore be carried out before general commercial distribution to a healthy population is undertaken. Obviously, high-risk patients will not be so critical in their acceptance of such vaccines.

The case for an annual large-scale inoculation in a healthy industrial population has still not been proven, and indeed at the present stage of develop- 
ment a decreasing response may well be met in successive years. But the case for further development by the pharmaceutical firms is strong, as is the need for more clinical trials.
REFERENCES Howell, R. W., and Stott, A. N. B. (1964). Brit. J. industr. Med.,

Meichen, F. W., Rogan, E., and Howell, R. W. (1962). Ibid., $19,203$.

Richardson, I. M., and Kilpatrick, S. J. (1964). Med. Off., 111, 5. 